\title{
Clinical Utility of Weekly Laboratory Testing in the Outpatient Management of Preeclampsia and Gestational Hypertension
}

\author{
John A. Morgan, MD ${ }^{1}$ Lauren E. McCalmont, MD ${ }^{1}$ Craig V. Towers, MD ${ }^{2}$ Melissa Davis, MD ${ }^{2}$ \\ Miriam Hankins, MD ${ }^{1}$ Niyati Rangnekar, BS ${ }^{2}$ Mary Ellen McNeal, BSN ${ }^{2}$ David F. Lewis, MD ${ }^{1}$
}

\footnotetext{
${ }^{1}$ Department of Obstetrics and Gynecology, LSU Health Shreveport, Shreveport, Louisiana

${ }^{2}$ Department of Obstetrics and Gynecology, The University of

Tennessee Health Science Center, Knoxville, Tennessee
}

Address for correspondence John Andrew Morgan, MD, Department of Obstetrics and Gynecology, LSU Health Shreveport, 1501 Kings Highway, Shreveport, LA 71130 (e-mail: jmorg1@Isuhsc.edu).

Am J Perinatol Rep 2020;10:e62-e67.

\author{
Abstract \\ Keywords \\ - mild preeclampsia \\ - preeclampsia without \\ severe features \\ - gestational \\ hypertension \\ - chronic hypertension \\ in pregnancy \\ - superimposed \\ preeclampsia \\ - liver enzymes \\ - platelet count \\ - preeclampsia with \\ severe features
}

Objective To investigate the utility of obtaining weekly laboratory testing in patients managed as an outpatient for gestational hypertension and preeclampsia without severe features.

Study Design A multisite retrospective cohort study was performed evaluating preterm women diagnosed with gestational hypertension/preeclampsia managed in an outpatient setting between gestational ages of $23^{0 / 7}$ and $36^{6 / 7}$. Patients were divided into two groups: weekly laboratory evaluation (laboratories group) and a no laboratories group. The primary study outcome was composite maternal morbidity including more than one of the following: development of severe features, HELLP syndrome, eclampsia, placental abruption, maternal intensive care unit admission, or maternal death.

Results A total of 204 patients were included in this study, laboratories group $(n=120)$ and no laboratories group $(n=84)$. The laboratories group was older (28.8 vs. 26.6 years, $p=0.02$ ), had a higher rate of chronic hypertension (44 [36.7\%] vs. 17 [20.2\%], $p=0.01$ ), and more often experienced the primary composite outcome (53 [44.2\%] vs. 24 [28.5\%], $p=0.02$ ). No patients in our cohort were delivered for abnormal laboratory values.

Conclusion This study found that weekly laboratory testing may have minimal clinical utility in the outpatient management protocol in monitoring patients with mild gestational hypertension or preeclampsia. Delivery was guided by other clinical factors.
The safety and cost effectiveness of the outpatient management of gestational hypertension and preeclampsia without severe features has been validated in multiple studies and is currently supported by the American College of Obstetricians and Gynecologists (ACOG) ${ }^{1-4}$ Prerequisites for outpatient management include reasonable assurance of patient willingness to comply with frequent maternal and fetal evaluation and maternal understanding of warning symptoms of preeclampsia with severe features. ${ }^{1}$

Current recommendations for outpatient management of gestational hypertension and preeclampsia without severe features include at least one clinic visit each week. ${ }^{1}$ Maternal evaluation should include review of symptomatology associated with severe preeclampsia, blood pressure measurement, received

November 26, 2019

accepted

December 5, 2019
DOI https://doi.org/

$10.1055 / \mathrm{s}-0040-1702935$. ISSN 2157-6998.
Copyright $\odot 2020$ by Thieme Medical

Publishers, Inc., 333 Seventh Avenue, New York, NY 10001, USA. Tel: $+1(212) 760-0888$.
License terms

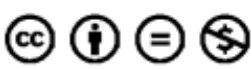


and weekly laboratory testing (platelet count, serum creatinine, and liver transaminases). ${ }^{1,4}$ The recommendation for weekly laboratory testing is based on expert opinion, with the goal of early detection of severe preeclampsia and associated end-organ damage. ${ }^{1,4}$

The utility of weekly laboratory testing in the outpatient management of preeclampsia is currently unclear and has not been investigated to date. The objective of the current study was to determine if there is an association between weekly laboratory testing for severe features of preeclampsia and decreased maternal morbidity in patients managed outpatient for gestational hypertension and preeclampsia without severe features.

\section{Materials and Methods}

This is a multicenter, retrospective, cohort study performed at two tertiary care teaching hospitals. All patients diagnosed with gestational hypertension, preeclampsia without severe features, and chronic hypertension with superimposed preeclampsia without severe features at LSU Health Shreveport, Shreveport, LA and The University of Tennessee Medical Center, Knoxville, TN between January 1, 2012, and August 18, 2018, were evaluated for inclusion.

The Institutional Review Board at the LSU Health Shreveport, Shreveport, LA approved the study, and The University of Tennessee Medical Center, Knoxville, TN obtained independent approval through their Institutional Review Board. International Classification of Diseases, Ninth and Tenth Revision codes were used to obtain a list of patients with the diagnosis of gestational hypertension, preeclampsia without severe features, or chronic hypertension without severe features. Linked neonatal charts were reviewed to obtain neonatal outcome data.

Patients were included in the final analysis if they had a singleton pregnancy between $23^{0 / 7}$ and $36^{6 / 7}$ weeks; were diagnosed with gestational hypertension, preeclampsia without severe features, or chronic hypertension with superimposed preeclampsia without severe features; and were treated as an outpatient following diagnosis. All patients at each study site which met the inclusion criteria during the study period were included in the study. Patients were excluded if they were treated inpatient from diagnosis until delivery, if they had a multiple gestation, and if they had preexisting liver or renal disease with abnormal baseline liver function or renal function laboratory values. Most patients in the study cohort were diagnosed during a short inpatient admission. During this brief inpatient stay, each patient was deemed a candidate for outpatient management by a maternal-fetal medicine specialist due to stability of hypertensive disorder and assessment of reliability of the patient to follow-up with twice weekly evaluation.

The primary outcome of the study was a composite of maternal morbidity including one or more of the following: severe features of preeclampsia at delivery, HELLP syndrome, placental abruption, eclampsia, maternal death, and maternal intensive care unit (ICU) admission. Secondary outcomes included indication for delivery (37 weeks, severe uncon- trolled maternal blood pressure, maternal symptomatology associated with severe preeclampsia, abnormal laboratory values, fetal indication, spontaneous labor, or other), gestational age at diagnosis, gestational age at delivery, latency from diagnosis to delivery, and compliance with scheduled outpatient visits.

As the focus of our study is the utilization of laboratory values, we understood the possibility of ascertainment bias during data collection for the indication for delivery. For this reason, an additional secondary outcome evaluated was the presence of abnormal laboratory values at delivery which was defined as thrombocytopenia (platelet count $<100,000 \times 10^{9} / \mathrm{L}$ ), impaired liver function (liver enzymes twice the upper limit of normal), and renal insufficiency (serum creatinine $1.1 \mathrm{mg} / \mathrm{dL}$ or doubling of baseline creatinine). As these numbers are objective measures of endorgan dysfunction, they are not subject to bias.

Patients were divided into two groups for comparison. The first group was managed outpatient with weekly laboratory testing (laboratories group) and the second group was managed outpatient without weekly laboratory testing (no laboratories group). ACOG published its Hypertension in Pregnancy Task Force Report in November 2013 which recommended weekly laboratory assessment in the outpatient management of hypertensive disorders of pregnancy. The majority of the no laboratories group was managed prior to this time, before weekly laboratory testing became standard of care, or throughout early 2014 before this practice had been fully adopted at the study sites.

The diagnosis of gestational hypertension, preeclampsia without severe features, or chronic hypertension with superimposed preeclampsia was based on ACOG criteria at the time of diagnosis at both sites. ${ }^{1}$ The current diagnostic criteria are as follows: Gestational hypertension is defined as systolic blood pressure more than $140 \mathrm{~mm} \mathrm{Hg}$ or diastolic blood pressure more than $90 \mathrm{~mm} \mathrm{Hg}$ on two separate occasions more than 4 hours apart without associated proteinuria or other signs of end-organ dysfunction. ${ }^{1}$ Preeclampsia without severe features criteria contains identical blood pressure criteria but with associated proteinuria defined as protein-creatinine ratio more than 0.3 or 24-hour urine protein more than $300 \mathrm{mg}$. $^{1}$ Chronic hypertension with superimposed preeclampsia is diagnosed with a sudden increase in blood pressure or proteinuria in a patient with preexisting hypertension. $^{5}$

Statistical analysis was performed using GraphPad Prism 7 (La Jolla, CA). All continuous data were compared using $t$ test and all categorical data were compared using chi-square or Fisher's exact test. A $p$-value of $<0.05$ was considered statistically significant. All tests were considered based on a two-sided alternative hypothesis.

\section{Results}

A total of 204 patients met inclusion criteria and are included in the final analysis: 120 patients in the laboratories group and 84 patients in the no laboratories group. The LSU Health Shreveport, Shreveport, LA contributed 100 patients, and 
e64 Utility of Routine Laboratory Testing in Mild Preeclampsia Morgan et al.

Table 1 Maternal characteristics of 204 patients with mild gestational hypertension, mild preeclampsia, and chronic hypertension with mild superimposed preeclampsia managed as an outpatient

\begin{tabular}{|l|l|l|l|}
\hline & Laboratories $(N=120)$ & No laboratories $(N=84)$ & $p$-Value \\
\hline Age $(\mathrm{y})$ & $28.8(24-34)$ & $26.6(22-31.7)$ & 0.02 \\
\hline Body mass index $\left(\mathrm{kg} / \mathrm{m}^{2}\right)$ & $35.7(29.3-41.1)$ & $34.9(27.2-40.9)$ & 0.51 \\
\hline White & $60(50)$ & $45(53.6)$ & 0.67 \\
\hline Black & $56(46.7)$ & $38(45.2)$ & 0.89 \\
\hline Hispanic & $4(3.3)$ & $1(1.2)$ & 0.65 \\
\hline Nulliparity & $35(29.2)$ & $21(25)$ & 0.53 \\
\hline Smoking & $22(18.3)$ & $17(22.1)$ & 0.86 \\
\hline History of preeclampsia & $33(27.5)$ & $23(27.3)$ & 0.99 \\
\hline On antihypertensive medications & $30 / 44(68.2)$ & $9 / 17(52.9)$ & 0.37 \\
\hline Total diabetes & $38(31.6)$ & $19(22.6)$ & 0.20 \\
\hline Pregestational diabetes & $18 / 38(47.4)$ & $9 / 19(47.4)$ & 0.99 \\
\hline Gestational diabetes & $20 / 38(52.6)$ & $10 / 19(52.6)$ & 0.99 \\
\hline Renal disease & $6(5)$ & $6(7.1)$ & 0.56 \\
\hline Intrauterine growth restriction & $19(15.8)$ & $8(9.5)$ & 0.19 \\
\hline Diagnosis & & & \\
\hline Gestational hypertension & $19(15.8)$ & $27(32.2)$ & 0.006 \\
\hline Preeclampsia without severe features & $57(47.5)$ & $40(47.6)$ & 0.99 \\
\hline cHTN with SIPE without severe features & $44(36.7)$ & $17(20.2)$ & 0.01 \\
\hline
\end{tabular}

Abbreviations: cHTN, chronic hypertension; SIPE, superimposed preeclampsia.

Note: Data are mean (interquartile range) or $n(\%)$.

The University of Tennessee Health Science Center, Knoxville, TN contributed 104 patients to the study cohort. Demographics between the two groups were compared and are shown in - Table 1. Patients in the laboratories group were older and more likely to have chronic hypertension. As the laboratories group had a significantly higher rate of chronic hypertension, this group also had a significantly higher rate of superimposed preeclampsia without severe features. Patients in the no laboratories group were more likely to have the diagnosis of gestational hypertension. All other demographic categories were similar between the two study groups.

Delivery outcomes were similar between the two groups with a few important differences (-Table 2). Significant findings were that the laboratories group had a mean gestational age at diagnosis that was 1 week earlier than the no

Table 2 Delivery information and neonatal outcomes of 204 patients with mild gestational hypertension, mild preeclampsia, and chronic hypertension with mild superimposed preeclampsia managed as an outpatient

\begin{tabular}{|l|l|l|l|}
\hline & Laboratories $(N=120)$ & No laboratories $(N=84)$ & $p$-Value \\
\hline GA at diagnosis (d) & $33^{0 / 7}\left(31^{2 / 7}-34^{6 / 7}\right)$ & $34^{0 / 7}\left(33^{0 / 7}-35^{2 / 7}\right)$ & 0.003 \\
\hline GA at delivery (wk) & $36^{2 / 7}\left(35^{5 / 7}-37^{1 / 7}\right)$ & $36^{6 / 7}\left(36^{0 / 7}-37^{3 / 7}\right)$ & 0.008 \\
\hline Latency (d) & $22.7(13-30)$ & $19.7(10-22.7)$ & 0.15 \\
\hline Spontaneous vaginal delivery & $44(36.7)$ & $34(40.5)$ & 0.66 \\
\hline Primary cesarean delivery & $40(33.3)$ & $25(29.7)$ & 0.65 \\
\hline Repeat cesarean delivery & $34(28.3)$ & $22(26.1)$ & 0.75 \\
\hline Operative vaginal delivery & $2(1.6)$ & $3(3.6)$ & 0.40 \\
\hline Birth weight (g) & $2,762(2,380-3,190)$ & $2,901(2,410-3,280)$ & 0.14 \\
\hline Stillbirth & $1(0.8)$ & $0(0)$ & 0.99 \\
\hline NICU admission & $41(34.2)$ & $17(20.2)$ & $1(1.2)$ \\
\hline 5-min Apgar score $<7$ & $3(2.5)$ & 0.03 \\
\hline
\end{tabular}

Abbreviations: GA, gestational age; NICU, neonatal intensive care unit.

Note: Data are mean (interquartile range), $n(\%)$. 
Table 3 Primary outcome: composite maternal morbidity of 204 patients with mild gestational hypertension, mild preeclampsia, and chronic hypertension with mild superimposed preeclampsia managed as an outpatient

\begin{tabular}{|c|c|c|c|}
\hline & Laboratories $(N=120)$ & No laboratories $(N=84)$ & $p$-Value \\
\hline Composite maternal morbidity & $53(44.2)$ & $24(28.5)$ & 0.02 \\
\hline \multicolumn{4}{|l|}{ Individual outcomes } \\
\hline Severe features at delivery & $53(44.2)$ & $24(28.5)$ & 0.02 \\
\hline HELLP syndrome & $0(0)$ & $0(0)$ & 1 \\
\hline Placental abruption & $0(0)$ & $0(0)$ & 1 \\
\hline Eclampsia & $0(0)$ & $0(0)$ & 1 \\
\hline Maternal death & $0(0)$ & $0(0)$ & 1 \\
\hline Maternal ICU admission & $0(0)$ & $0(0)$ & 1 \\
\hline
\end{tabular}

Abbreviation: ICU, intensive care unit.

laboratories group and they also had a mean gestational age at delivery that was 4 days earlier than the no laboratories group. This explains the higher rate of neonatal intensive care unit (NICU) admission in the laboratories group. The groups had similar lengths of latency from diagnosis to delivery, however. Compliance with outpatient visits was similar between the two groups, and overall compliance was 83.7\%. Mode of delivery, birth weight, and 5-minute Apgar score $<7$ were also similar between the two groups. There was one stillbirth in the study cohort, which was in the laboratories group in a patient with preeclampsia without severe features who maintained $100 \%$ compliance with her follow-up visits.

Primary outcome data are shown in - Table 3. The primary outcome of composite maternal morbidity was more common in the laboratories group than the no laboratories group. A total of 53 patients in the laboratories group (44.2\%) versus 24 patients in the no laboratories group (28.5\%) experienced the primary outcome $(p=0.02)$. Analysis of the individual outcomes included in the composite morbidity revealed that the only outcome experienced in either group was the presence of severe features at delivery. No patient in the 204-patient cohort experienced HELLP syndrome, placental abruption, eclampsia, maternal death, or maternal ICU admission.

Indications for delivery were similar between the two groups with no statistically significant differences (-Fig. 1). The most common indication for delivery in both groups was reaching 37 weeks, the gestational age at which delivery is recommended by ACOG for gestational hypertension,

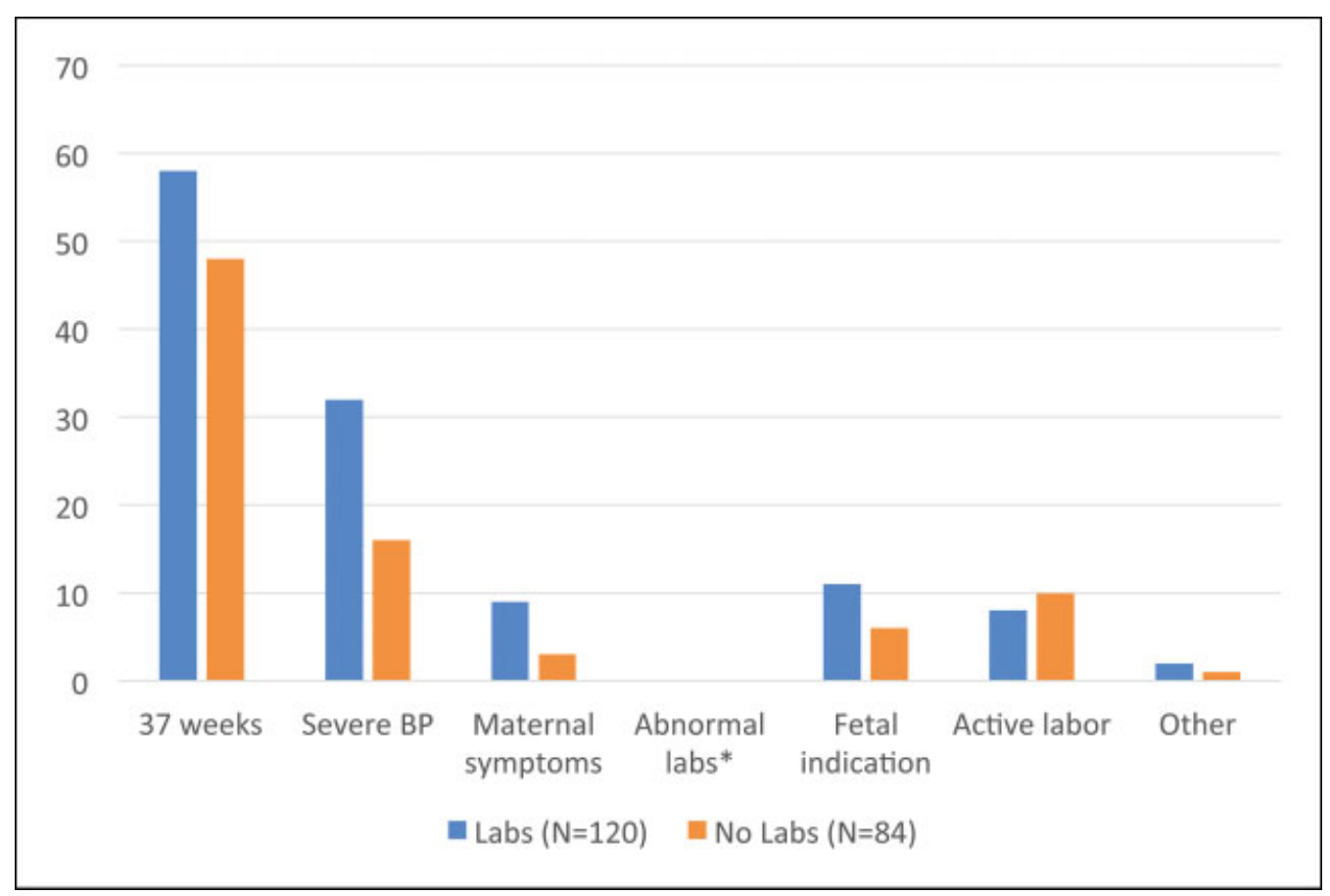

Fig. 1 Indications for delivery in 204 patients managed as an outpatient for mild gestational hypertension: weekly laboratory group versus no laboratory group. BP, blood pressure; labs, laboratory values. *Abnormal laboratory values defined as platelet count $<100,000 \times 109 / \mathrm{L}$, liver transaminases twice the upper limit of normal, and serum creatinine $1.1 \mathrm{mg} / \mathrm{dL}$ or doubling of baseline creatinine. No statistically significant differences between the two groups with regard to any indication for delivery (defined as $p<0.05$ ). 
preeclampsia without severe features, and chronic hypertension with superimposed preeclampsia without severe features. ${ }^{1,4}$ No patient in the study cohort was delivered for the indication of abnormal laboratory values as defined.

The presence of abnormal laboratory values at delivery was evaluated as an additional secondary outcome. Only one patient in the study cohort had abnormal laboratory values at delivery. This patient had preeclampsia without severe features and was seen in antenatal testing clinic where she was scheduled to receive a biophysical profile, have her blood pressure checked, and be evaluated by a maternal-fetal medicine specialist. On intake, she was noted to have a severe range blood pressure, defined as $>160 \mathrm{~mm} \mathrm{Hg}$ systolic. On her clinical assessment, she also reported a severe headache, unresponsive to medication she had taken at home prior to her presentation. She was diagnosed with preeclampsia with severe features and immediately sent to the labor and delivery unit for assessment and subsequent delivery. Her admission laboratory values showed thrombocytopenia with platelet count of $<100,000 \times 10^{9} / \mathrm{L}$ with normal liver transaminases and creatinine. For this particular patient, her severe blood pressure and symptomatology were the primary indications for delivery with thrombocytopenia being an incidental finding.

Due to the demographic differences, as well as the differences in the composite outcome between the two groups, we performed an additional secondary outcome investigating the differences in latency period and the development of severe features according to each individual hypertensive disorder. Mean latency was slightly longer in the gestational hypertension group (24.4 days) compared with preeclampsia without severe features (20.5 days) and chronic hypertension with superimposed preeclampsia without severe features (20.9 days). The development of severe features, however, was twice as likely in the group with chronic hypertension with superimposed preeclampsia without severe features ( 37 of 61 patients, $60.7 \%$ ) compared with either gestational hypertension (10 of 46 patients, $21.7 \%$ ) or preeclampsia without severe features (30 of 97 patients, $30.9 \%$ ), $p<0.001$ for both comparisons. The development of severe features in the gestational hypertension group compared with the preeclampsia without severe features group was not different, $p=0.25$.

\section{Discussion}

Our multicenter, retrospective study is the first to investigate the clinical utility of weekly laboratory testing in the outpatient management of gestational hypertension and preeclampsia without severe features to our knowledge. Our extensive literature review spanning PubMed, Medline, Google Scholar, and Embase did not reveal any prior studies specifically investigating this topic. The comparison of patients who were managed without laboratory testing to those managed with laboratory testing showed that the group managed without laboratory testing did not have less favorable maternal or fetal outcomes. In our 204-patient cohort, no patient was delivered primarily due to an abnor- mality in laboratory values, and only 1 patient had abnormal laboratories at the time of delivery (thrombocytopenia only).

Patients in the laboratories group were older and had higher rates of chronic hypertension, which subsequently led to findings of higher rates of superimposed preeclampsia without severe features, earlier age at both diagnosis and delivery, as well as higher rates of NICU admission. Patients in our cohort with the diagnosis of chronic hypertension with superimposed preeclampsia had twice the rate of development of severe features compared with preeclampsia without severe features or gestational hypertension. Additionally, prior studies support that chronic hypertension in pregnancy has been associated with a higher risk of both maternal and fetal morbidity and mortality. The laboratories group is a higher risk population than the no laboratories group in our comparison, so the differences in outcomes reported is not surprising. ${ }^{5}$

The laboratories group was found to have a higher rate of the composite maternal morbidity, but the only outcome noted in either group of the study cohort was the development of severe features. No patient developed HELLP syndrome, placental abruption, eclampsia, maternal death, or maternal ICU admission. In our cohort, the decision to treat each patient as an outpatient was made at the discretion of a maternal-fetal medicine specialist. Our overall compliance with outpatient management was high at $83.7 \%$. Additionally, the majority of patients included in our study were diagnosed with hypertensive disorders after 30 weeks of gestation. Our original study design was set to include patients diagnosed as early as $23^{0 / 7}$ weeks, but only 16 of the patients in our cohort were diagnosed at less than 30 weeks. Patients with multiple risk factors for preeclampsia are more likely to develop early onset and/or severe features of preeclampsia. ${ }^{6}$ Our study cohort self-selected to include mostly patients diagnosed with hypertensive disorders at a later gestational age. Patients diagnosed in the third trimester were more likely to be viewed as appropriate for management in the outpatient setting. Our study findings may not be applicable to patients diagnosed in the second trimester. We hypothesize that our appropriate selection of patients to be treated outpatient, close monitoring, and high patient compliance allowed early recognition of severe signs and symptoms of preeclampsia with subsequent delivery prior to the development of less favorable outcomes included in the composite.

While the laboratories group had a higher rate of development of severe features, this group also had a higher rate of chronic hypertension. Physiologic changes in pregnancy can cause blood pressure increases in the late second and early third trimesters in patients with chronic hypertension. ${ }^{7}$ It is often difficult to distinguish between physiologic elevations in blood pressure and the development of superimposed preeclampsia. When a patient's blood pressures are persistently in the severe range, they are often treated as preeclampsia with severe features despite the absence of other severe signs or symptoms. ${ }^{5,7}$ Maternal headache refractory to medication is another diagnostic criterion for preeclampsia with severe features, but it is also a common complaint in 
pregnancy. ${ }^{8}$ Headache as an isolated symptom of preeclampsia with severe features has not been associated with adverse outcomes in the literature. ${ }^{8}$ Physicians being aware of increased risk of adverse outcomes with superimposed preeclampsia are more likely to act on increasing blood pressure or maternal symptoms in these individuals. ${ }^{5}$ The laboratories group had a $7 \%$ higher reported rate of severe blood pressures and a $4 \%$ higher rate of maternal symptomatology prompting delivery, which could explain the higher rate of severe features in the laboratories group.

Indications for delivery were similar between the two groups. The most significant finding in our study cohort is that no patient was delivered for the primary indication of abnormal laboratory values. Schoen et al reported in a cohort of 198 patients with superimposed preeclampsia treated outpatient, $16(8.1 \%)$ of patients were delivered for the primary indication of abnormal laboratory values. ${ }^{3}$ As previously discussed, superimposed preeclampsia carries a higher risk of progression to severe disease. Our population had an overall lower number of patients with superimposed preeclampsia and a higher rate of preeclampsia without severe features and gestational hypertension, so our overall study population is relatively lower risk and would be expected to have a lower rate of laboratory abnormalities. Valent et al reported a similar rate of $9.5 \%$ of patients being delivered for abnormal laboratory values, but this cohort only included patients managed inpatient which is not comparable to our relatively lower risk cohort. ${ }^{9}$ We strengthened our reporting of delivery indication by also reporting that only one patient in our cohort had abnormal laboratory values at delivery.

Strengths of this study include a large number of patients managed at two tertiary teaching hospitals with an even racial distribution which makes the results generalizable. Major medical conditions were not excluded unless baseline signs of end-organ dysfunction were present. As the population effected by hypertensive disorders of pregnancy generally has multiple medical comorbidities, this also makes the results generalizable. The main weakness of this study is the retrospective, nonrandomized study design. Over the course of the study period, the recommendations for management of hypertensive disorders as well as diagnostic criteria changed, ${ }^{1,4}$ and the physicians at the study sites became more comfortable treating these hypertensive disorders outpatient. A prospective trial at these two sites would likely yield a much higher number of patients over a similar time period.

We do not advise a change in the current standard of care regarding weekly laboratory testing in the outpatient management of gestational hypertension, preeclampsia without severe features, and superimposed preeclampsia without severe features based on our findings. Our study does suggest that in a carefully selected, compliant patient population, the clinical evaluation regarding maternal blood pressure and symptomatology was used more often in the management of these patients. Elevated maternal blood pressure and/or maternal symptomatology associated with severe preeclampsia may be present before laboratory findings of end-organ dysfunction in this group. Our study findings also suggest that the diagnosis of chronic hypertension with superimposed preeclampsia is a higher risk group and may warrant more intensive follow-up than the diagnoses of preeclampsia without severe features and gestational hypertension.

In conclusion, our study suggests that weekly laboratory testing may have minimal clinical utility in the outpatient management of hypertensive disorders of pregnancy. Larger, prospective randomized trials are needed to determine the appropriate timing and frequency of laboratory testing in this population with a specific focus on stratifying the need for laboratories based on the particular hypertensive disorder that is diagnosed.

\section{Disclosure}

All authors report no conflicts of interest and have no financial disclosures to report.

\section{Note}

Oral presentation at the 86th Annual Meeting of the Central Association of Obstetricians and Gynecologists, October 19, 2019.

\section{Funding}

No sources of funding were used for this study.

\section{References}

1 ACOG Practice Bulletin No. 202: gestational hypertension and preeclampsia. Obstet Gynecol 2019;133(01):e1-e25

2 Barton JR, Istwan NB, Rhea D, Collins A, Stanziano GJ. Cost-savings analysis of an outpatient management program for women with pregnancy-related hypertensive conditions. Dis Manag 2006;9 (04):236-241

3 Schoen CN, Moreno SC, Saccone G, et al. Outpatient versus inpatient management for superimposed preeclampsia without severe features: a retrospective, multicenter study. J Matern Fetal Neonatal Med 2018;31(15):1993-1999

4 Sibai BM. Diagnosis and management of gestational hypertension and preeclampsia. Obstet Gynecol 2003;102(01):181-192

5 ACOG Practice Bulletin No. 203: chronic hypertension in pregnancy. Obstet Gynecol 2019;133(01):e26-e50

6 Wright D, Nicolaides KH. Aspirin delays the development of preeclampsia. Am J Obstet Gynecol 2019;220(06):580.e1-580.e6

7 Becker DA, Machemehl HC, Biggio JR, Siegel AM, Tita AT, Harper LM. Pregnancy outcomes of exacerbated chronic hypertension compared with superimposed preeclampsia. Am J Perinatol 2019; 36(08):872-878

8 Sperling JD, Dahlke JD, Huber WJ, Sibai BM. The role of headache in the classification and management of hypertensive disorders in pregnancy. Obstet Gynecol 2015;126(02):297-302

9 Valent AM, DeFranco EA, Allison A, et al. Expectant management of mild preeclampsia versus superimposed preeclampsia up to 37 weeks. Am J Obstet Gynecol 2015;212(04):515.e1-515.e8 